\title{
On the origin of the "cumulative semantic inhibition" effect
}

\author{
F.-Xavier Alario and Fermín Moscoso del Prado Martín \\ Aix-Marseille Université and C.N.R.S., Marseille, France
}

\begin{abstract}
We investigated whether the cumulative semantic inhibition effect found by Howard, Nickels, Coltheart, and Cole-Virtue (2006) provides information about semantic representations. By applying more sensitive statistical analyses to the original data set, we found a significant variation in the magnitude of the effect across categories. This variation cannot be explained by the naming speed of each category. In addition, using a subsample of the data, a second cumulative effect arouse for newly defined supracategories, over and above the effect of the original ones. We discuss these findings in terms of the representations that drive lexical access and show that they favor featural or distributed hypotheses.
\end{abstract}

In a recent article, Howard, Nickels, Coltheart, and Cole-Virtue (2006) reported a remarkable observation concerning lexical access during speech production. They asked speakers to name pictures of common objects presented in a continuous series of trials. The sequence of trials had an underlying structure; the members of a given category were separated by a variable, yet carefully controlled, number of trials. Such a design allowed a comparison between effects driven by the ordinal position of every response within its category and effects driven by the distance (in trials) between related items. ${ }^{1}$ The remarkable observation was that the ordinal position within the category had a very systematic effect, whereas the distance of the previous related item (measured in trials) did not affect performance. The relationship between ordinal position and average naming latency was reported to be linear. A unit increase in ordinal position led to an average increase of $30 \mathrm{msec}$ in naming latency $(95 \% \mathrm{CI}= \pm 8.2 \mathrm{msec})$. As is shown in their Figure 1, the average latency data, plotted against ordinal position, are remarkably aligned.

In the theoretical discussion of this phenomenon, Howard et al. (2006) focused on the cumulative property of the effect. They rightly highlighted that none of the current models of lexical access in language production predicts this observation. They then constructed a working model of word selection involving a process of priming and competitive selection, which simulates the observed cumulative effect. The purpose of the present article is not to address the cumulative nature of this effect (for a modelization of these findings that builds on errordriven learning, see Oppenheim, Dell, \& Schwartz, 2007, 2009; see also Navarrete, Mahon, \& Caramazza, 2008). Rather, we focus on the relatively undiscussed structure of the semantic representations. ${ }^{2}$ Howard et al.'s compu- tational model includes nondecomposed localist semantic representations for each item. A model variant including decomposed semantic features was also tested. These alternative semantic representations are motivated by a previous general hypothesis adopted in models of language production and are not explored in detail. The analysis Howard et al. presented suggests that both the localist and the distributed models are equally supported by the experimental data.

Our main question is whether the cumulative inhibition effect can be used to constrain theories of semantic representation. As we will show, the data reported by Howard et al. (2006) are in fact inconsistent with localist categorical representations. Our analysis also shows how this effect could be used to assess the structure of distributed representations.

Howard et al. (2006) used a total of 24 categories (listed in their Appendix). The use of such a large number of categories ensured a high statistical power for the analysis, in which the ordinal position and distance parameters were contrasted. It also led to considerable diversity in the definitions, with categories ranging from rather general sets (e.g., buildings or furniture) to more specific ensembles (e.g., computer equipment or farm animals). Despite this diversity in the definition of categories, the reported semantic cumulative effect is remarkably strong in the analysis of categories, suggesting that it is not driven by a subset of the materials.

In our investigation, we paid special attention to the role played by these categories in the observation of the effect. We will proceed in two steps, in which we will answer the following related questions: (1) Is the magnitude of the effect similar across the 24 categories, or does it show a systematic variability? (2) Is the effect better understood in terms of categorical representations of these particular

F.-X. Alario, francois-xavier.alario@univ-provence.fr 
categories or in terms of a by-product of some other representational structure?

To answer the first question, we reanalyzed Howard et al.'s (2006) original data set using a more sensitive technique. To answer the second question, we extended this analysis with newly defined categories replacing the original ones. Our analyses were based on the mixed-effect modeling methodology (Bates, 2005) recently introduced in psycholinguistics (Baayen, 2008; Baayen, Davidson, \& Bates, 2008). This technique relies on single-trial data, rather than on averages over participants, categories, or ordinal positions. This methodological choice yields two distinct benefits. First, it avoids an important shortcoming of the statistical analyses reported in Howard et al. As they acknowledged, there is a potential confound between ordinal position in the category and trial position in the experiment. The analysis performed to address this issue runs a risk of circularity. The effect of trial position was estimated on the data set and reinjected as a corrective parameter in the same data set. This procedure may have distorted the results. Second, the additional benefit of the new method is that it allows one to explicitly model the fixed versus random nature of the different effects under consideration. As is shown below, this enables more detailed and robust analyses than are possible with the traditional analysis techniques.

\section{VARIABILITY OF THE CUMULATIVE INHIBITION EFFECT ACROSS CATEGORIES}

By focusing on single-trial data, we were able to test, in a single analysis, the potential contributions of the different order and distance parameters that characterize every trial of the experiment. Furthermore, this analysis enabled the investigation of a question that was not addressed in the original study - namely, possible systematic variations in the magnitude of the cumulative inhibition effect across categories.

\section{Linear Analysis of the Data Set}

We obtained the data set used in the original study. This data set comprised a total of 2,568 trials (after the exclusion of filler trials, errors, and responses identified as outliers). The individual naming latencies were log transformed using the natural logarithm to reduce skewness and to approach a normal distribution, then were fitted to three linear models with different combinations of fixed, random, and mixed effects. The predictors were added se- quentially into these three models in the order in which they are mentioned below.

The first model, referred to as Hh-model 1 (for Howard's hypothesis modeling), was intended to test the same hypothesis as did Howard et al. (2006) - namely, that there is a main linear effect of ordinal position in the category, independent of the lag between related trials and of the position of the trials in the experiment. We defined as fixed effects the factors of theoretical interest-namely, the ordinal position in the category, the lag between the current trial and the previous trial from the same category, and trial number (i.e., the ordinal position in the experiment). In addition, to be able to handle trial-level data, participant and item identity were explicitly included as random effects in the model. As can be seen in Table 1, ordinal position had a significant inhibitory effect, and no evidence was found for influences of lag or trial number. In Hh-model 2, the effect of trial number was allowed to vary between participants (see Howard et al., 2006). This was done by including an interaction between the fixed effect of trial number and the random effect of participants (i.e., a mixed effect). A formal comparison of Hh-models 1 and 2-namely, a log-likelihood test - shows a significant improvement in the model's fit $\left[\chi^{2}(1)=13.6, p=.001\right]$, whereas the estimates for the theoretically relevant predictors remain largely unaffected. Together, these results are fully concordant with those of Howard et al.

More interesting, in the third model (Hh-model 3), we further estimated the cross-categorical variability of the linear cumulative semantic inhibition effect. To do so, we included categories as an additional random effect, on top of participants and items. This new random effect had 24 levels under which the items were nested. We computed its main random effect, as well as its interaction with the inhibitory fixed effect of ordinal position (i.e., a mixed effect). The main effect indicates possible systematic contributions of the categories over and above the items that compose them. The interaction with ordinal position provides an estimate of the systematic variation in the amount of cumulative semantic inhibition produced specifically by each category.

The comparison of the models' estimates in Table 1 indicates that the magnitude and significance of the main linear semantic effect was largely unaffected by the inclusion of this new random factor. A formal comparison between models by means of a log-likelihood test shows a significant improvement of the model's fit, both from the inclusion of a variable intercept across categories $\left[\chi^{2}(1)=\right.$

Table 1

Comparison of the Fixed Effects in Linear Mixed-Effects Models of the Log-Transformed Naming Latency in the Full Data Set (2,568 Observations From 24 Participants Naming 120 Items in 24 Categories)

\begin{tabular}{|c|c|c|c|c|c|c|c|c|c|}
\hline \multirow[b]{3}{*}{ Fixed Effect } & \multicolumn{9}{|c|}{ Hh-Model } \\
\hline & \multicolumn{3}{|c|}{1} & \multicolumn{3}{|c|}{2} & \multicolumn{3}{|c|}{3} \\
\hline & $\beta$ & $t$ & $p$ & $\beta$ & $t$ & $p$ & $\beta$ & $t$ & $p$ \\
\hline Ordinal position & $3.81 \cdot 10^{-2}$ & 7.22 & $<.001$ & $3.80 \cdot 10^{-2}$ & 7.25 & $<.001$ & $3.87 \cdot 10^{-2}$ & 5.51 & $<.001$ \\
\hline Lag & $5.18 \cdot 10^{-4}$ & 0.20 & .84 & $6.59 \cdot 10^{-4}$ & 0.26 & .80 & $7.16 \cdot 10^{-4}$ & 0.28 & .78 \\
\hline Trial number & $2.26 \cdot 10^{-4}$ & 1.55 & .12 & $2.17 \cdot 10^{-4}$ & 0.97 & .33 & $2.09 \cdot 10^{-4}$ & 0.94 & .35 \\
\hline
\end{tabular}

Note-Hh-model, Howard's hypothesis modeling. $\beta$ is in log scale, and $d f=2564$ for al $t$ tests. 
13.0, $p<.001]$ and from a variable ordinal-position effect across categories $\left[\chi^{2}(1)=16.5, p<.001\right]$. This indicates that, although it is true that there was a main inhibitory effect of the ordinal position within the category, valid on average for all categories, two additional factors not reported by Howard et al. (2006) need to be considered. On one hand, there was a significant random effect of category, meaning that items in some categories were systematically faster than items in other categories. On the other hand, the amount of inhibition provided by each occurrence of an item within a category showed significant systematic variation across individual categories: Some categories produced consistently more inhibition than did others.

To clarify this finding, the main random effect of categories and its interaction with ordinal positions are plotted in Figure 1A. Three findings are noteworthy. First, the overall speed for each category presented a considerable degree of variability, which was estimated to be over and above item variability. Second, although all of the coefficients were positive, there was also significant variability in the magnitude of the linear cumulative effect across categories. This indicates that every single category contributed to the cumulative inhibition effect (with the possible exception of Category 8, body parts). Finally, the main speed of a category was unrelated to its contribution to the semantic cumulative effect, with no evidence for a correlation between the two estimates $[r=.15, t(22)=0.69, p=.49]$.

\section{Discussion}

The results of the analysis that we performed over the original data set provides a confirmation of the observations made by Howard et al. (2006) on the basis of data averaged over participants, items, and categories. The results also show that the linear cumulative semantic inhibition effect was present for all of the categories that were tested (with one possible exception). In other words, the cumulative semantic inhibition effect was in no way a consequence of the trial positions of successive members of a category or a consequence of averaging across a heterogeneous data set. In addition, two new issues have arisen from our analyses. First, there was a systematic variation in the overall speeds of items belonging to different categories. Second, and more important, there was also systematic variation in the strength of the cumulative semantic inhibition across categories. Finally, the overall speed with which the members of a category were named was unrelated to the strength of the cumulative inhibition shown by that category. Therefore, we cannot conclude that the variability in the size of the cumulative effect was a mere consequence of variations in naming speed. One possible origin of the variation in the cumulative inhibition effect across categories may be the relationships among the items that compose the categories. The theoretical interpretation of these findings will be addressed in more detail in the General Discussion section. For now, these observations set the stage for a deeper exploration of the effect on a subset of the data.

\section{CONTRASTIVE HYPOTHESIS ABOUT THE CUMULATIVE INHIBITION EFFECT}

In the second analysis that we report, we investigated the representational status of some of the categories used in Howard et al. (2006). Our first analysis showed that all
A

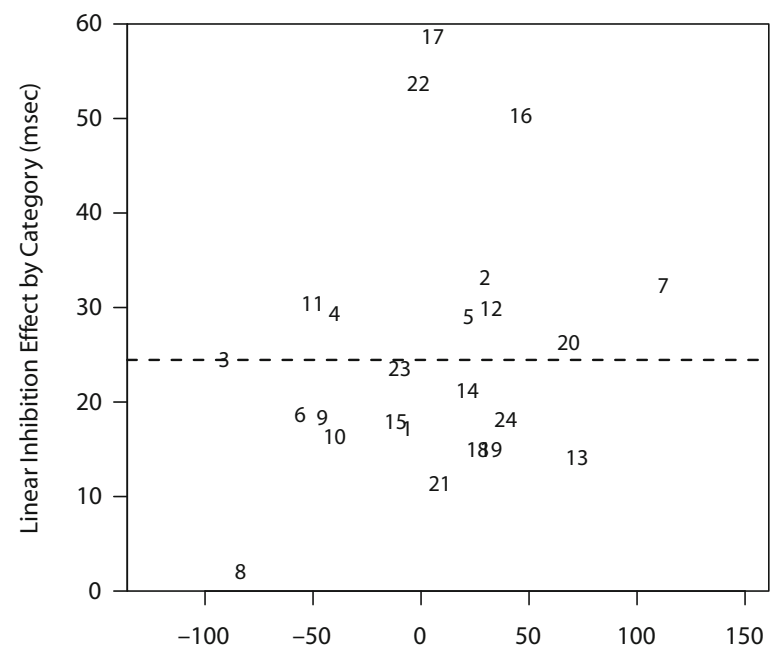

B

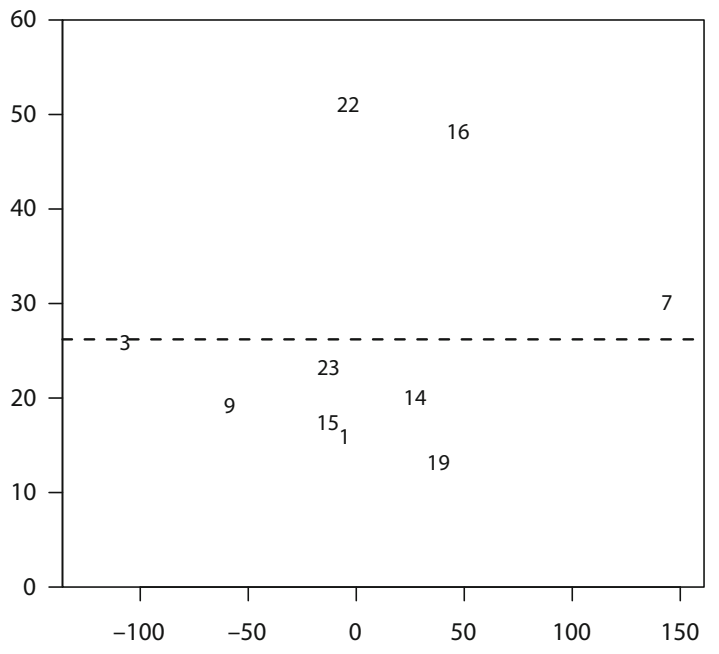

Main Random Effect of Category (msec)

Figure 1. Estimates of the random intercept of category ( $x$-axis) and the mixed interaction between category and ordinal position $(y$-axis) for each of the original categories. Larger values in the $x$-axis indicate slower categories. Larger values in the $y$-axis indicate stronger coefficients for the linear semantic effect (i.e., stronger inhibition). Each data point is a category (see the Appendix for details). (A) Complete data set (Hh-model 2). (B) Subset used in the second analysis (N-model 1). The magnitudes and relative orderings of these random effect estimates have been validated using a bootstrapping technique $(N=100$; Efron, 1979). 
of the categories produced a sizable cumulative inhibition effect. Does this mean that the categories should be understood, in any strong sense, as representational categories in the speakers' cognitive system? Although a positive answer to that question would be rather surprising (e.g., zoo and farm animals were distinguished; white goods was a category in itself), the question is a useful anchor point for our rationale.

An inspection of the list of materials shows that at least 10 categories allow a natural regrouping in pairs of cocategories under a supracategory (see Table 2). This property allows manipulation of the structure of the categories used to analyze the data, and, hence, it provides answers to the question above. If two cocategories are representationally independent, naming the members of one of them should not affect how the members of the other are named any more than would having named any other items. For example, the speed of naming farm animals should be independent of whether zoo animals (or buildings) were named before. By contrast, if the items of cocategories share part of their representation, such influence should be apparent in the naming performance. In the latter case, the relative contributions of supra- and cocategory groupings might be clarified.

We report a series of analyses on a subset of the data from Howard at al. (2006), in which only the materials listed in Table 2 were included. We proceed in three steps. First, we show that the original effect of interest is similarly present in the restricted data set. Second, we show that having named the cocategory earlier in the experiment affects naming latencies in a systematic fashion. Finally, we contrast two assumptions about the underlying representations that may cause the influence of cocategories on one another.

\section{Step 1: Is the Cumulative Inhibition Effect Present in the Restricted Data Set?}

The data from the 10 categories in Table 2 were entered in a linear regression model very similar ${ }^{3}$ to Hh-model 3. This model is summarized under N-model 1 (for new hypothesis modeling) in Table 3 . The linear inhibition effect driven by ordinal position was also present in this restricted data set, with an estimated size of the same order of mag- nitude as that in the complete data set. This conclusion is strengthened by an inspection of Figures $1 \mathrm{~A}$ and $1 \mathrm{~B}$, which show that individual estimates of the inhibition effect for each of the categories in the restricted data set (Figure 1B) are very similar to those obtained previously from the complete data set (Figure 1A). Here, again, there was a significant improvement of the model's fit with the inclusion of a variable intercept across categories $\left[\chi^{2}(1)=5.99, p=\right.$ $.014]$ and a variable ordinal position effect $\left[\chi^{2}(1)=5.08\right.$, $p=.025]$. The estimate of N-model 1 for the ordinal position effect is plotted in Figure 2A.

In contrast to the full-data-set analyses, trial position produced a significant effect in the restricted data set of $\mathrm{N}$-model 1. This result will be discussed below in relation to the manipulations concerning the relative positions of cocategories. For the rest, the restricted data set provides a robust and safe testing ground for the hypotheses stated above about the mutual influence of cocategories on the cumulative inhibition effect.

\section{Step 2: Is There a Dependency \\ Between Cocategories?}

The pairs of cocategories summarized in Table 2 may or may not be subserved by common representations. If two cocategories are independent, naming the items of one of them should be independent of whether the members of the cocategory have been named before. By contrast, if two cocategories have a common underlying representation, responses to the category that is presented second should be slowed down by the previous naming of a member of its cocategory. We tested this prediction by differentiating cocategories on the basis of the order in which they were named in the experiment. For this test to be possible, two precautions needed to be taken.

First, the order in which the categories were presented across participants had to be checked. The last column of Table 2 summarizes the relative position of each of the two cocategories. The order in which they were presented in the experimental lists is relatively well balanced across participants. Furthermore, in most cases, the two cocategories did not overlap in the stimulus sequence. Any effect observed for cocategories presented second can therefore be attributed to their position in the experi-

Table 2

Regrouping of a Subset of the Original Items in Supracategories

\begin{tabular}{|c|c|c|c|c|}
\hline Cocategories & Members & Supracategory & Motivation & Number \\
\hline $\begin{array}{l}\text { Farm animals } \\
\text { Zoo animals }\end{array}$ & $\begin{array}{l}\text { cow, donkey, horse, pig, sheep } \\
\text { gorilla, monkey, hippo, tiger, elephant }\end{array}$ & Mammals & All are mammals & $\begin{array}{l}10 \\
14\end{array}$ \\
\hline $\begin{array}{l}\text { Clothes } \\
\text { Headgear }\end{array}$ & $\begin{array}{l}\text { bra, jacket, pajamas, skirt, sock } \\
\text { beret, cap, crown, hat, helmet }\end{array}$ & Clothing & All items are worn & $\begin{array}{l}11 \\
13\end{array}$ \\
\hline $\begin{array}{l}\text { Fish } \\
\text { Shellfish }\end{array}$ & $\begin{array}{l}\text { eel, goldfish, shark, stingray, swordfish } \\
\text { crab, lobster, mussel, oyster, prawn }\end{array}$ & Sea creatures & All live in the sea & $\begin{array}{l}14 \\
10\end{array}$ \\
\hline $\begin{array}{l}\text { Computer equipment } \\
\text { Audio-visual equipment }\end{array}$ & $\begin{array}{l}\text { computer, joystick, keyboard, mouse, printer } \\
\text { headphones, microphone, radio, speaker, TV }\end{array}$ & Electronic equipment & $\begin{array}{l}\text { We watch TV on computers, } \\
\text { and we browse networks on TVs }\end{array}$ & $\begin{array}{l}13 \\
11\end{array}$ \\
\hline $\begin{array}{l}\text { Fruits } \\
\text { Vegetables }\end{array}$ & $\begin{array}{l}\text { apple, banana, lemon, pear, orange } \\
\text { broccoli, carrot, cauliflower, onion, potato }\end{array}$ & Fruits and vegetables & Never dissociated in patients ${ }^{\mathrm{a}}$ & $\begin{array}{l}14 \\
10\end{array}$ \\
\hline
\end{tabular}

Note-Number, the number of participants $(N=24)$ who named each category before its cocategory. aCapitani, Laiacona, Mahon, and Caramazza (2003). 
A

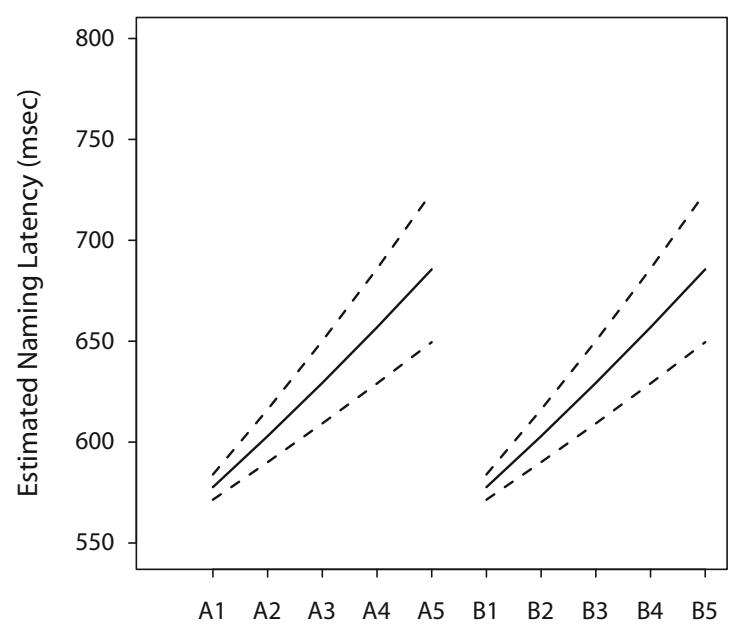

\section{B Original Categories and Supracategories}

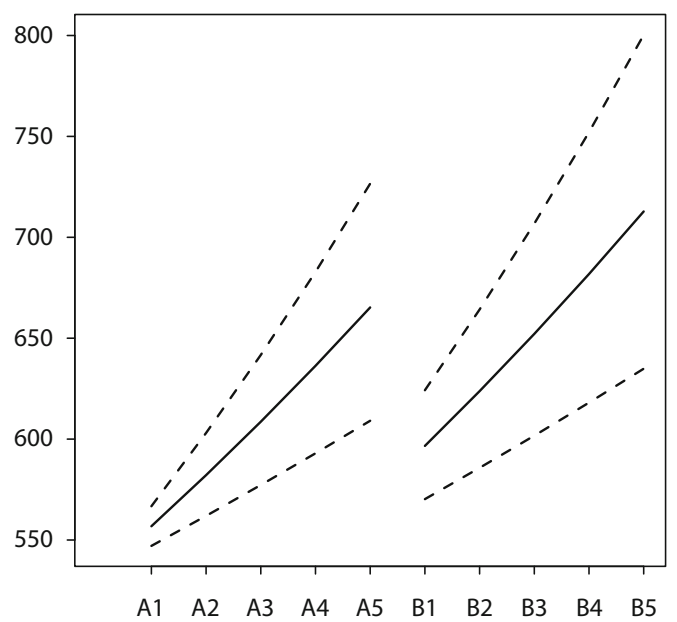

C Supracategories

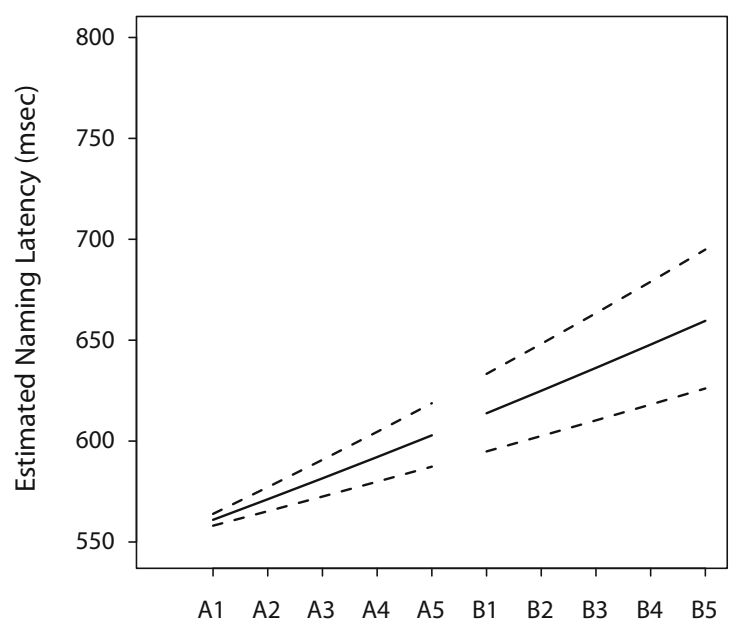

Figure 2. Estimated performance, with standard errors, for the members of two cocategories (A and B, shown on the $x$-axis) in different models instantiating different hypotheses. A rationale for choosing among models is described in the General Discussion section. (A) Both cocategories are independent; their ordinal positions produce independent significant effects (N-model 1). (B) The cocategories depend on one another; both the ordinal position in the original categories and in the supracategory produce independent significant effects (N-model 5). (C) The cocategories are in fact a single supracategory; ordinal position only within the supracategory produces a significant effect (model not reported).

mental list, rather than to the specifics of the items that compose them.

Second, the absolute position of each cocategory within each experimental list had to be controlled. Obviously, the second cocategory was always presented later in the experiment. If there is a systematic variation of performance with trial position (see, e.g., the suggestive effect of trial position in N-model 1 in Table 3), it should be taken into account when interpreting relative category position. This was done by considering three related predictors. The first one, used previously, was the position of the trials in the list. The second predictor was the absolute position of the category within the experimental list. It was defined as a constant number for all the members of a cocategorynamely, the trial number of the first item of the category. Finally, we introduced the critical predictor of the relative position of two cocategories, with values 1 (vs. 0) when the cocategory had (vs. had not) been named before. We also included the predictor of theoretical interest: ordinal position within the cocategory.

$\mathrm{N}$-model 2 in Table 3 shows that the effect of ordinal position within the category was significant. More impor- 
Table 3

Comparison of Estimated Parameters in Various Linear Regression

Models of the Log-Transformed Naming Latency in the Restricted Data Set (1,056 Observations From 24 Participants Naming 50 Items in 10 Categories)

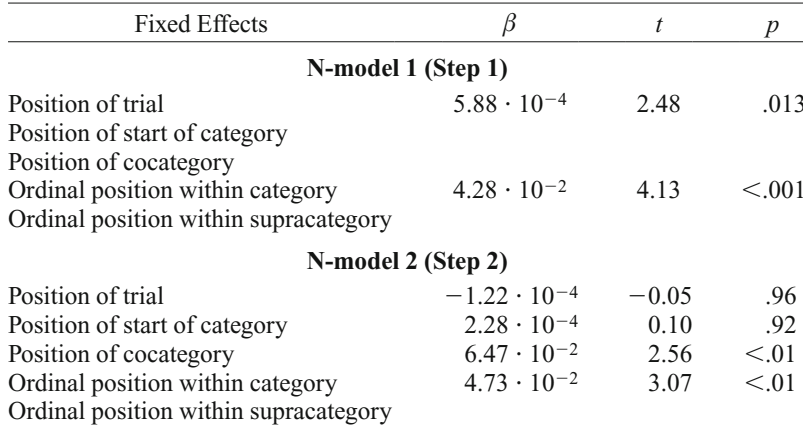

N-model 3 (Step 2)

Position of trial

Position of start of category

Position of cocategory

Ordinal position within category

$7.00 \cdot 10^{-2} \quad 3.56 \quad<.001$

Ordinal position within supracategory

$4.68 \cdot 10^{-2} \quad 4.49<.001$

N-model 4 (Step 3)

\begin{tabular}{|c|c|c|c|}
\hline \multirow{2}{*}{\multicolumn{4}{|c|}{$\begin{array}{l}\text { Position of trial } \\
\text { Position of start of category }\end{array}$}} \\
\hline & & & \\
\hline Ordinal position within category & $3.07 \cdot 10^{-2}$ & 2.61 & $<.01$ \\
\hline Ordinal position within supracategory & $1.38 \cdot 10^{-2}$ & 2.54 & .01 \\
\hline \multicolumn{4}{|c|}{ N-model 5 (Step 3) } \\
\hline $\begin{array}{l}\text { Position of trial } \\
\text { Position of start of category } \\
\text { Position of cocategory }\end{array}$ & & & \\
\hline Ordinal position within category & $3.02 \cdot 10^{-2}$ & 2.61 & $<.001$ \\
\hline Ordinal position within supracategory & $1.47 \cdot 10^{-2}$ & 3.55 & $<.001$ \\
\hline
\end{tabular}

tant, the relative position of the cocategory had a strong and significant effect, whereas neither the absolute position of the trial nor the absolute position of the category was significant. This result indicates that the relative position of the cocategory was indeed the best predictor of naming latencies - better than trial position or category position. A secondary analysis clarified that this result was not due to trial position being a less efficient predictor than a category's relative position. We re-paired the cocategories randomly in five pairs 2,000 times. Of those, 257 sample lists contained only unrelated cocategory pairs. For these 257 samples, we estimated the effects of trial position and relative position given the new pairings. Trial position produced a significant effect $(t>1.65, p<.05)$ for $73 \%$ of the samples; an unrelated category's relative position was significant for only $7.4 \%$ of the samples, and in all cases the estimated relative position effect went in the opposite direction (second category faster) to what we observed in $\mathrm{N}$-model 2 with properly related pairs (see Table 3).

This finding also clarifies why trial position may have been significant in the restricted data set (N-model 1) and not in the complete data set (e.g., Hh-model 2). The restricted data set included only categories whose sec- ond occurrence was slowed down by their previously presented cocategory. By contrast, the complete data set included many categories that did not have a cocategory named earlier.

Summing up, this second analysis reveals a clear dependency between cocategories that is not due to the absolute position of the trials in the experiment and that is observed over and above the effect of ordinal position within a category. This is summarized in N-model 3 in Table 3, where only significant variables were included. The occurrence of the second cocategory was slower than the first one, by an order of magnitude that was similar to the effect of ordinal position within a category. The variable magnitude of the cumulative inhibition effect did not reach significance in these two models. Whether this result is a mere consequence of the reduction in power of the analysis or a reflection of a theoretically meaningful observation remains an open question.

A very simple explanation for the dependency between cocategories would be that cocategories are in fact a single category - namely, the supracategory listed in Table 2. In this view, going from the end of one cocategory (e.g., the fifth farm animal) to the beginning of its cocategory (e.g., the first zoo animal) is equivalent to increasing by one 
the ordinal position of the item in the corresponding supracategory. If this were an appropriate description of the data, the cumulative inhibition effect should make trials at the beginning of the second cocategory slower than trials at the end of the first cocategory. A quick approximating test of this hypothesis (data set size $=215$ trials) shows, if anything, the opposite pattern. The first trial of the second cocategory was faster than the fifth trial of the first cocategory $[t(212)=-2.15, p=.033]$, irrespective of their intertrial lag $[t(212)<1]$. This finding suggests that the effect of the relative positions of cocategories may be more than a simple accumulation of inhibition within a supracategory.

\section{Step 3: Are Cocategories Better Described As a Single Encompassing Category?}

In this third step, we tested more formally whether the two cocategories could be functionally reduced to a single supracategory. This account makes a clear prediction. Ordinal position within the supracategory (range: 1-10) should produce a significant effect that should absorb the effect of ordinal position within the (original) category (range: 1-5). A representation of the prediction of such a model is plotted in Figure 2C. Alternatively, the slowing down of the cocategory occurring second may not be because of the items' belonging to a single category. The underlying representation of items that share a single feature or property within and across cocategories may be responsible for the effect. An example of such a feature for the cocategories farm and zoo animals could be four-limbed animal or mammal. This alternative account also makes a clear prediction. Ordinal position within the supracategory (range: 1-10) should produce a significant effect in addition to the previously reported effect of ordinal position within the (original) category (range: 1-5).

These alternative predictions were tested in N-models 4 and 5 , reported in Table 3 . The estimates of N-model 4 show that both the ordinal position within the supracategory and the ordinal position within the original category have independent significant inhibitory effects. The effect of supracategory position cannot be ascribed to trial position, which was also included in the model. N-model 5 reports the estimates when only the significant variables are considered in the model. ${ }^{4}$ The combined estimated effects of ordinal position within the cocategory and ordinal positions within the supracategory are plotted in Figure 2B. In summary, this third step establishes that the ordinal position within the supracategory has a significant inhibitory effect over and above a significant effect of ordinal position within the (original) category.

\section{Discussion}

The analysis of single-trial data using linear mixedeffects models has equipped us with sufficient statistical power to analyze a subset of the original data from Howard et al. (2006) with models similar to those used for the complete data set. These analyses are based on the plausible pairings of the categories summarized in Table 2. They indicate that, at least for the items under consideration, the semantic cumulative inhibition effect is not restricted within categories.

The plots in Figures 2A and 2C illustrate the cumulative inhibition effect for models that consider a single level of category (respectively, when only the cocategory or only the supracategory level is considered). Figure $2 \mathrm{~B}$ plots the model where the dependency between cocategories is captured by both predictors. Our analyses enable us to discard both single-category models in terms of their predictive accuracy. The predictions are significantly better when both representational levels are considered.

Note that statistics alone are not sufficient to disentangle the two models where cocategories depend on one another (namely, the second and third steps in Table 3), since they both produce equally good predictions for this data set. One way to resolve this issue would be to consider a hypothetical experiment where cocategories would be intermixed. The definition and predictions of the model with two ordinal factors at the two levels are straightforward, and our interpretation of the results described above leads to a distinct prediction. Figure 3B should be the better description of the results, over and above Figures $3 \mathrm{~A}$ and $3 \mathrm{C}$. The prediction depicted in Figure 3B is an ordinal inhibition effect between items of any cocategory plus an ordinal inhibition effect of a different magnitude between successive trials belonging to two different cocategories. In contrast, defining which cocategory comes first when cocategories are intermixed in the experiment would require additional assumptions. For this reason, the model with two ordinal factors may be preferred. Pending further evidence, however, we will simply draw the important conclusion that the dependency between cocategories is not reducible to the supracategory.

\section{GENERAL DISCUSSION}

The cumulative inhibition effect reported by Howard et al. (2006) is present across categories. This confirms the robustness of their finding when random variation is explicitly taken into account and when the relative contributions of ordinal position in the category and trial position in the experiment are considered simultaneously. On top of this, our analyses have added some facts that were not previously considered. We found a significant variation in the magnitude of the cumulative effect. This variation is independent of the variation in overall speed across categories. In the restricted data set, a further cumulative effect was found for newly defined supracategories, over and above the original ordinal position effect within cocategories. These deeper analyses and new findings allow contrasting theories of semantic representation, so that we can better understand the involvement of these representations in lexical access.

From a methodological perspective, our analyses argue in favor of using single-trial information, instead of averaging performance across levels. This is especially true in the case at hand, where noncounterbalanceable absolute and relative positions of individual trials have to be taken into consideration simultaneously to understand the data. 
A

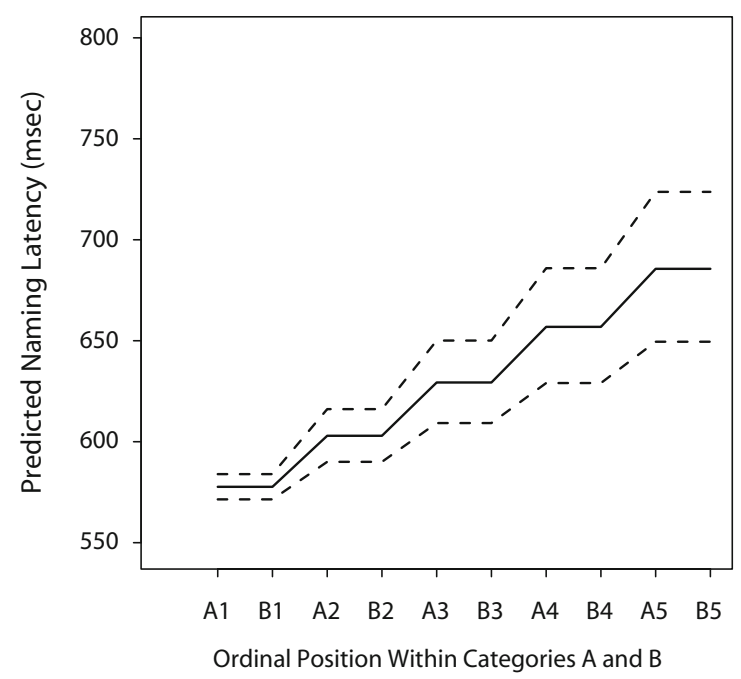

\section{B Original Categories and Supracategories}

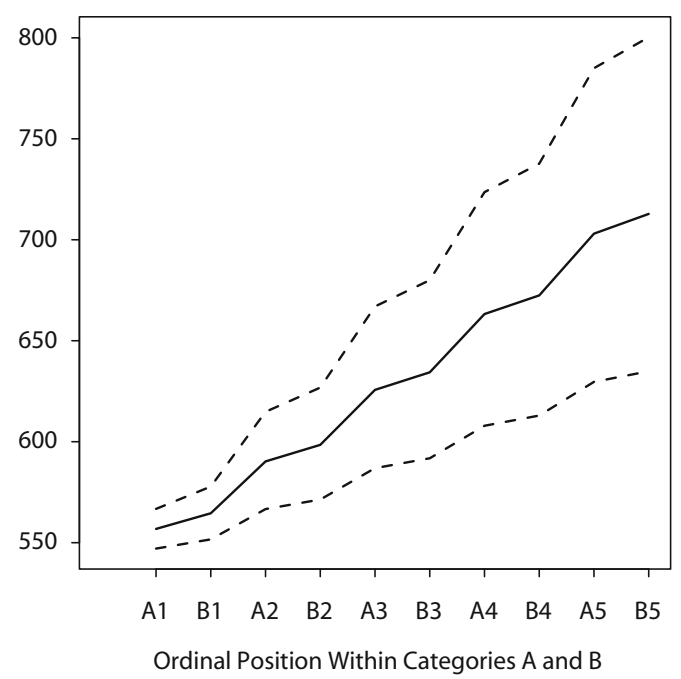

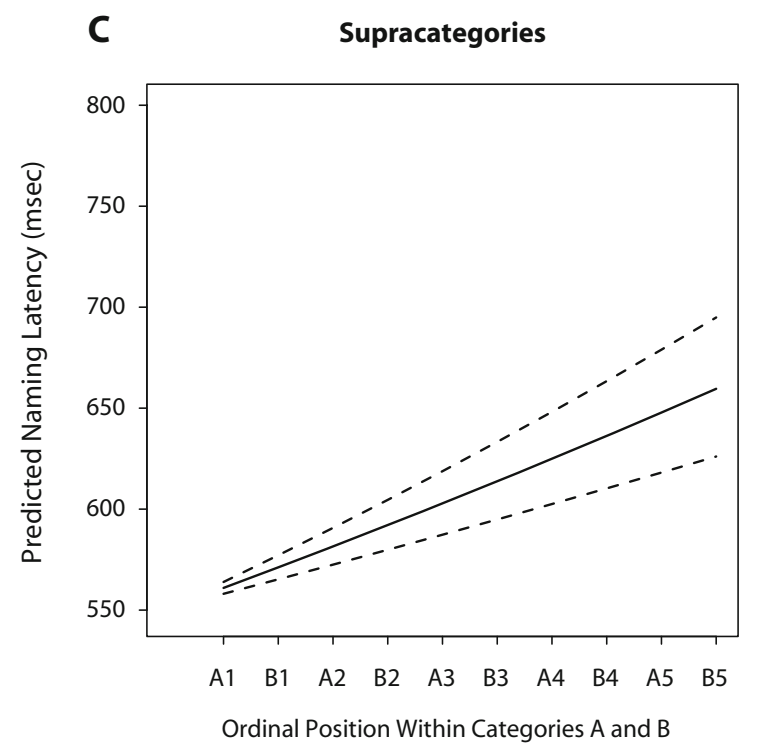

Figure 3. Predicted performance, with standard errors, for the members of two cocategories (A and B, shown on the $x$-axis) interleaved in the experimental lists for different models instantiating different hypotheses. (A) Both cocategories are independent. (B) The cocategories depend on one another, such that both the ordinal position in the original (Howard et al., 2006) categories and in the supracategory produce independent significant effects. (C) The cocategories are in fact a single supracategory, such that ordinal position only within the supracategory produces a significant effect.

Thus, our first conclusion is methodological. We have shown how the mixed-effect methodology that Baayen et al. (2008) introduced for language comprehension studies also provides a powerful tool for language production studies.

From a theoretical perspective, the results of our analyses enrich the conclusions of Howard et al. (2006) in several ways. The first important observation is the sig- nificantly variable inhibition effect across categories. The fact that it is unrelated to overall speed indicates that this variation does not reflect a mere performance effect. One plausible explanation for this variation may come from some intrinsic property (or properties) of the categories in question. Our analysis was conducted post hoc; hence, such properties were not manipulated explicitly. However, several dimensions come to mind that have been previ- 
ously proposed to differentially characterize the relationships between members of different categories: structural similarity across the members of a category (Humphreys, Riddoch, \& Quinlan, 1988), degree of correlation of features across the category (Caramazza, Hillis, Rapp, \& Romani, 1990; McRae, de Sa, \& Seidenberg, 1997; Moss, Tyler, \& Taylor, 2007), semantic distance within and across categories (Vigliocco, Vinson, Lewis, \& Garrett, 2004), and so on. Testing the link between one or several of these dimensions to the amount of semantic cumulative inhibition would be a useful tool for contrasting these hypotheses. In previous research, the effect of semantic distance has been tested with other picture-naming paradigms. Some authors have reported worse performance when the target and context words were semantically closer (Vigliocco, Vinson, Damian, \& Levelt, 2002). Others, however, have observed that reducing semantic distance (or typicality) had no effect (Lupker, 1979) or had a facilitatory effect (Mahon, Costa, Peterson, Vargas, $\&$ Caramazza, 2007). Further ad hoc studies in which the simple picture-naming task employed by Howard et al. (2006) was used may contribute to the clarification of the relative contributions of these parameters and their role in the cumulative inhibition effect.

The second important observation is the fact that more than one grouping parameter (i.e., category) is needed to account for the performance. As was shown by our second analysis, a categorical representation of the items that relies solely on one level of abstraction - be it the level of cocategories or the level of supracategories - would not capture the whole pattern of cumulative inhibition. This fact supports a hierarchical representation in which individual items belong to more than one semantic (or structural) nesting level. It excludes strict localist hypotheses, such as those implemented in the working model proposed by Howard et al. (2006) or other versions. It may not exclude, however, more sophisticated implementations of localist representations whose connection weights or numbers code for properties elsewhere termed features (e.g., Page, 2000). In these cases, the difference between localist and distributed representations is not straightforward. For instance, Moss et al. (2007) and Vigliocco and Vinson (2007) noted that most proposals now favor featural or distributed representations in one way or another (Lambon Ralph, McClelland, Patterson, Galton, \& Hodges, 2001; McClelland \& Rogers, 2003; McRae et al., 1997). This being said, the analysis that we report here was constrained by the categories available in Howard et al., yielding only two nesting levels that were statistically tractable. Yet this was sufficient to indicate that the cumulative inhibition effect is a useful tool for testing the structure of the representational network involved in lexical access. Testing whether more than two levels of abstraction ultimately modulate performance should contribute to the clarification of the hierarchical organization of the representations driving lexical access.

One final point should be raised about the nature of the cumulative inhibition effect. Following Howard et al. (2006), we have modeled the effect with linear predic- tors (we did not observe any nonlinear components that reached significance). The positive correlation between mean response times and the corresponding variance across conditions makes it impossible to model the data as arising from a linear additive model. It is for this reason that we log transformed the data prior to our analysis. Note that this transformation implies a nonlinear, nonadditive effect in the natural scale. This would seem to contrast with Howard et al.'s original finding of a linear additive trend. The limited range of variation of the observed values makes these two alternatives virtually indistinguishable (the range in the log-transformed data was 5.53-7.58). However, we would expect to observe deviations from linearity in data sets with wider ranges of ordinal category positions (i.e., above 5). In other words, a linear effect with a magnitude of $26 \mathrm{msec}$ may become unrealistic for item groupings comprising 10 or more items. The apparently linear effect may prove to be nonlinear after all.

In conclusion, we reported an investigation of the cumulative semantic inhibition effect reported by Howard et al. (2006). Where Howard et al. reported a single regression line, we showed that a richness of systematic variations can be observed and - more important - predicted. These variations are better understood in terms of featural or distributed representations driving lexical access. Our analysis further shows how these variations and predictions can be fruitfully used to confront current theories of semantic representation on a quantitative basis.

\section{AUTHOR NOTE}

This work was supported by Grant ANR-07-JCJC-0074 from the Agence Nationale de la Recherche (France). The authors thank David Howard for kindly providing the complete data set of the original publication and two anonymous reviewers for useful comments. Correspondence concerning this article should be addressed to F.-X. Alario, Laboratoire de Psychologie Cognitive, CNRS \& Aix-Marseille Université, Centre St Charles, Bât. 9, Case D, 3 place Victor Hugo, 13331 Marseille Cedex 3, France (e-mail: francois-xavier.alario@univ-provence.fr).

\section{REFERENCES}

BaAyen, R. H. (2008). Analyzing linguistic data: A practical introduction to statistics using $R$. Cambridge: Cambridge University Press.

BaAyen, R. H., Davidson, D. J., \& Bates, D. M. (2008). Mixed-effects modeling with crossed random effects for subjects and items. Journal of Memory \& Language, 59, 390-412.

BATES, D. M. (2005). Fitting linear mixed models in R. R News, 5, 2730.

Capitani, E., Laiacona, M., Mahon, B. Z., \& Caramazza, A. (2003). What are the facts of category-specific deficits? A critical review of the clinical evidence. Cognitive Neuropsychology, 20, 213-261.

Caramazza, A., Hillis, A. E., Rapp, B. C., \& Romani, C. E. (1990). The multiple semantics hypothesis: Multiple confusions? Cognitive Neuropsychology, 7, 161-189.

EFron, B. (1979). Bootstrap methods: Another look at the jackknife. Annals of Statistics, 7, 1-26.

Howard, D., Nickels, L., Coltheart, M., \& Cole-Virtue, J. (2006). Cumulative semantic inhibition in picture naming: Experimental and computational studies. Cognition, 100, 464-482.

Humphreys, G. W., Riddoch, M. J., \& Quinlan, P. T. (1988). Cascade processes in picture identification. Cognitive Neuropsychology, $\mathbf{5}, 67-103$

Lambon Ralph, M. A., McClelland, J. L., Patterson, K., Galton, C. J., \& Hodges, J. R. (2001). No right to speak? The relationship between object naming and semantic impairment: Neuropsychological 
evidence and a computational model. Journal of Cognitive Neuroscience, 13, 341-356.

LUPKER, S. J. (1979). The semantic nature of response competition in the picture-word interference task. Memory \& Cognition, 7, 485-495.

Mahon, B. Z., Costa, A., Peterson, R., Vargas, K. A., \& CaraMAZZA, A. (2007). Lexical selection is not by competition: A reinterpretation of semantic interference and facilitation effects in the picture-word interference paradigm. Journal of Experimental Psychology: Learning, Memory, \& Cognition, 33, 503-535.

McClelland, J. L., \& Rogers, T. T. (2003). The parallel distributed processing approach to semantic cognition. Nature Reviews Neuroscience, 4, 310-322.

McRae, K., de SA, V., \& Seidenberg, M. (1997). On the nature and scope of feature representations of word meaning. Journal of Experimental Psychology: General, 126, 99-130.

Moss, H. E., TyLER, L. K., \& TAYLOR, K. I. (2007). Conceptual structure. In M. G. Gaskell (Ed.), The Oxford handbook of psycholinguistics (pp. 217-234). Oxford: Oxford University Press.

Navarrete, E., Mahon, B. Z., \& Caramazza, A. (2008, September). The cumulative semantic cost in picture naming [Il costo cumulativo intracategoriale in compiti di denominazio-ne di figure]. Paper presented at the XIVth Congresso Nazionale Associazione Italiana di Psicologia, Padua.

Oppenheim, G., Dell, G., \& Schwartz, M. (2007). Cumulative semantic interference as learning. Brain \& Language, 103, 175-176.

Oppenheim, G., Dell, G., \& Schwartz, M. (2009). The dark side of incremental learning: A model of cumulative semantic interference during lexical access in speech production. Manuscript submitted for publication.
PAGE, M. (2000). Connectionist modelling in psychology: A localist manifesto. Behavioral \& Brain Sciences, 23, 443-467.

Vigliocco, G., \& Vinson, D. P. (2007). Semantic representation. In M. G. Gaskell (Ed.), The Oxford handbook of psycholinguistics (pp. 195-215). Oxford: Oxford University Press.

Vigliocco, G., Vinson, D. P., Damian, M. F., \& Levelt, W. J. M. (2002). Semantic distance effects on object and action naming. Cognition, 85, B61-B69.

Vigliocco, G., Vinson, D. P., Lewis, W., \& Garrett, M. F. (2004). The meaning of object and action words. Cognitive Psychology, $\mathbf{4 8}$, 422-488.

\section{NOTES}

1. More specifically, each list comprised 120 items drawn from 24 different categories (i.e., 5 items per category), plus a set of 45 filler pictures. The items of a given category were separated by two, four, six, or eight trials; this arrangement was counterbalanced across participants and categories.

2. For consistency with the terminology used in Howard et al. (2006), we will refer to the effect as cumulative semantic inhibition. A possible contribution of visual factors will be mentioned in the General Discussion section.

3. The lag between trials and the lag between cocategories variables were included in previous versions of the analysis. They never contributed significantly and, hence, are omitted here for simplicity.

4. As was the case in Step 2, the variable magnitude of the cumulative inhibition effect did not reach significance in this analysis.

\section{APPENDIX}

Names of the Categories in Howard et al. (2006)

\begin{tabular}{llll}
\hline 1. zoo animals & 7. fish & 13. house parts & 19. vegetables \\
2. birds & 8. body parts & 14. computer equipment & 20. buildings \\
3. fruits & 9. clothes & 15. farm animals & 21. celestial phenomena \\
4. musical instruments & 10. tableware & 16. shellfish & 22. headgear \\
5. tools & 11. furniture & 17. white goods & 23. audio-visual \\
6. transport & 12. bugs & 18. reptiles and amphibians & 24. landscape features \\
\hline
\end{tabular}

Note-The numbers are those used in Figures 1A and 1B. For details on the category members, see Howard et al. (2006). 